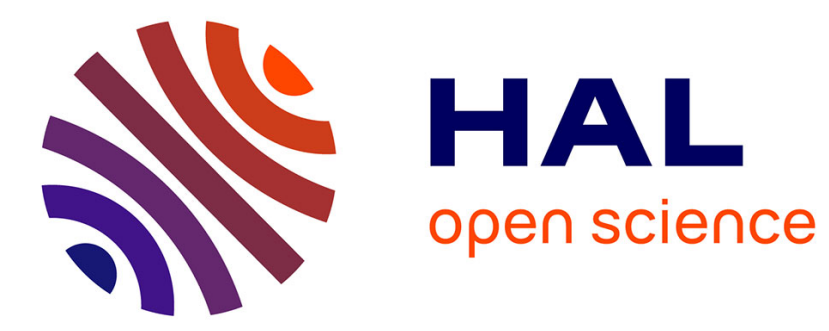

\title{
Troubles dans la relation de personne
}

Frank Wagner

\section{To cite this version:}

Frank Wagner. Troubles dans la relation de personne. Poétique: revue de théorie et d'analyse littéraire, 2015, 178 (2), pp.275-296. 10.3917/poeti.178.0275 . hal-02460326

\section{HAL Id: hal-02460326 \\ https://hal.univ-rennes2.fr/hal-02460326}

Submitted on 30 Jan 2020

HAL is a multi-disciplinary open access archive for the deposit and dissemination of scientific research documents, whether they are published or not. The documents may come from teaching and research institutions in France or abroad, or from public or private research centers.
L'archive ouverte pluridisciplinaire HAL, est destinée au dépôt et à la diffusion de documents scientifiques de niveau recherche, publiés ou non, émanant des établissements d'enseignement et de recherche français ou étrangers, des laboratoires publics ou privés. 


\title{
TROUBLES DANS LA RELATION DE PERSONNE
}

\author{
Frank Wagner
}

Le Seuil | « Poétique »

2015/2 n 178 | pages 275 à 296

ISSN $1245-1274$

ISBN 9782021219500

Article disponible en ligne à l'adresse :

https://www.cairn.info/revue-poetique-2015-2-page-275.htm

Distribution électronique Cairn.info pour Le Seuil.

(C) Le Seuil. Tous droits réservés pour tous pays.

La reproduction ou représentation de cet article, notamment par photocopie, n'est autorisée que dans les limites des conditions générales d'utilisation du site ou, le cas échéant, des conditions générales de la licence souscrite par votre établissement. Toute autre reproduction ou représentation, en tout ou partie, sous quelque forme et de quelque manière que ce soit, est interdite sauf accord préalable et écrit de l'éditeur, en dehors des cas prévus par la législation en vigueur en France. Il est précisé que son stockage dans une base de données est également interdit. 


\title{
RELECTURE \\ Frank Wagner Troubles dans la relation de personne
}

\author{
" "Qui dit ça, maman?”" \\ André Gide, Journal.
}

"Alfred Bruyas [...] m’affirme que, selon lui, tous les “il”, en littérature, sont des "je" déguisés, de même que tous les "je" sont des "il" en puissance."

Jean-Philippe Toussaint, «L'Ile des anamorphoses».

\section{Pour quelques pages de plus...}

Dans La Vérité sur Marie, roman publié en $2009^{1}$, Jean-Philippe Toussaint délègue à un narrateur extra-homodiégétique (j'y reviens dans un instant) l'évocation de "L'Ile des anamorphoses, cette nouvelle apocryphe de Borges, où l'écrivain qui invente la troisième personne en littérature finit, au terme d'un long processus de dépérissement solipsiste, déprimé et vaincu, par renoncer à son invention et se remet à écrire à la première personne» (p. 168). Ce bref apologue narrativisé de même que ses prolongements ${ }^{2}$, d'où provient la seconde épigraphe ci-dessus,

1. Aux éditions de Minuit.

2. Le passage cité a donné lieu à un concours de nouvelles, à l'occasion duquel il s'agissait d'écrire la «nouvelle apocryphe de Borges» évoquée dans La Vérité sur Marie. Les textes des lauréats ont été publiés sur le site électronique de Jean-Philippe Toussaint, et peuvent être consultés en suivant le lien suivant: http://www.jptoussaint.com/borges-projet.html

C'est la version de Jean-Philippe Toussaint (p. 2-3) qui m’a fourni la seconde épigraphe de cet article. 
peuvent constituer un indice parmi d'autres de l'intérêt persistant - souvenons-nous de Kafka, Proust et James - des écrivains pour les questions de «personne»; ainsi qu'une incitation à réexaminer la notion de relation de personne, telle que l'a formalisée Gérard Genette.

Sans doute l'intérêt de la chose ne paraîtra-t-il guère évident à ceux qui, plus encore aujourd'hui qu'hier, à l'austérité du régime narratologique préfèrent des "nourritures plus aphrodisiaques ${ }^{3}$ "; sans doute la question a-t-elle en outre déjà fait couler beaucoup d'encre, au point d'avoir été jadis considérée comme «decidedly over worked " " S'il peut malgré tout valoir la peine de passer outre d'éventuelles réticences, c'est en raison des effets de flottement qui se manifestent en ces matières. Pour le dire autrement, cet article est né d'un trouble: suscité d'une part par certaines des définitions que Gérard Genette lui-même propose de la relation de personne; d'autre part, et à vrai dire surtout, par l'usage qu'en font d'autres théoriciens, hostiles ou non aux positions genettiennes - et où se fait jour pour le moins quelque risque de confusion, en particulier avec le paramètre du niveau narratif. Le désir d'endiguer de telles dérives confusionnistes, préjudiciables aux analyses qu'elles informent, suffit donc à justifier cet effort de clarification, puisque «mon» trouble est, consciemment ou non, à l'évidence partagé.

Aussi conviendra-t-il tout d'abord d'examiner avec autant d'attention que possible les diverses formulations qui permettent à Genette, de Figures II à Nouveau Discours $d u$ récit, en "passant» bien sûr par Figures $I I I^{6}$ et Palimpsestes" ${ }^{7}$, de formaliser la nature de la relation qu'entretient l'instance narrative du récit fictionnel à l'histoire qu'elle raconte. Les enseignements de cette enquête "archéologique» favoriseront ensuite l'examen des divers problèmes théoriques que peut susciter la conception genettienne de la relation de personne, qu'il s'agisse de l'articulation du choix grammatical et de l'attitude narrative, du lien déjà évoqué à la question du niveau, du problème corollaire des interventions narratoriales, ou encore du statut ontologique du narrateur, comme du statut logique de l'activité linguistique qui préside à son apparition ${ }^{8}$. A toutes fins utiles, précisons que cette étape «méta-narratologique» se déploiera "sans haine, sans violence et sans arme ${ }^{9}$ ", en vertu notamment de l'intuition partagée qu'en régime fictionnel le narrateur existe (je l'ai moi aussi rencontré) - ce qui ne signifie pas pour autant que les arguments des tenants de l'hypothèse du «récit sans narrateur» seront par principe ignorés. Enfin, narratologie et plus généralement poétique du récit ne pouvant se satisfaire d'un fonctionnement autarcique, il s'agira d'examiner in fine comment nombre d'auteurs d'œuvres

3. Gérard Genette, Nouveau Discours du récit, Paris, éd. du Seuil, «Poétique», 1983, p. 7.

4. Ibid., p. 65.

5. Paris, éd. du Seuil, «Tel quel», 1969; «Points», 1979, pour l'édition citée.

6. Paris, éd. du Seuil, «Poétique», 1972.

7. Paris, éd. du Seuil, «Poétique», 1982.

8. Ce qui impliquera in fine l'examen des positions défendues par Genette dans Fiction et diction, Paris, éd. du Seuil, «Poétique», 1991.

9. Peut-être aura-t-on reconnu là le sous-titre des Egouts du paradis d'Albert Spaggiari (Paris, Albin Michel, 1978). Qu'on n'en infère pas pour autant que cet article constituerait, aux yeux de son auteur, l'équivalent narratologique du «casse du siècle»... 
contemporaines, disons des années 1960 à nos jours, non contents de problématiser la relation de personne dans leurs écrits, à la façon de Jean-Philippe Toussaint, la troublent de propos délibéré, au nom de la singularité de leurs projets artistiques respectifs - ce qui, pour le théoricien, peut constituer une incitation supplémentaire à porter un regard critique sur les outils qu'il élabore et entend utiliser à des fins d'analyse.

\section{Archéologie: de la «diégèse» et de ses préfixes}

Pour mémoire, dans l' «Essai de méthode» que constitue le «Discours du récit» de Figures III, au chapitre "Voix", après avoir fermement établi la distinction principielle des instances narrative et littéraire, ainsi que de leurs situations respectives («[...] la situation narrative d'un récit de fiction ne se ramène jamais à sa situation d'écriture", p. 226), Genette procède à l'étude successive des temps de la narration (antériorité, simultanéité ou postériorité par rapport au temps de l'histoire), des niveaux narratifs (extra-, intra-, métadiégétique) et de la transgression de leurs frontières par voie de métalepse, avant seulement d'envisager la "personne ${ }^{10}$ ». Quitte à revenir en arrière en cas de besoin, commençons par... la fin.

Si Genette se désolidarise de l'opposition usuelle entre «récit à la première personne» et "récit à la troisième personne», c'est, on s'en souvient, en vertu de la conviction que: «En tant que le narrateur peut à tout instant intervenir comme tel dans le récit, toute narration est, par définition, virtuellement faite à la première personne» (p. 252). Aussi est-ce conformément à cette prémisse qu'il affirme que "Le choix du romancier n'est pas entre deux formes grammaticales [“je" vs "il"], mais entre deux attitudes narratives (dont les formes grammaticales ne sont qu'une conséquence mécanique) : faire raconter l'histoire par l'un de ses "personnages", ou par un narrateur étranger à cette histoire» (ibidem); avant de proposer la typologie que l'on sait:

On distinguera donc ici deux types de récits: l'un à narrateur absent de l'histoire qu'il raconte (exemple: Homère dans l'Iliade, ou Flaubert dans L'Education sentimentale), l'autre à narrateur présent comme personnage dans l'histoire qu'il raconte (exemple: Gil Blas ou Wuthering Heights). Je nomme le premier type, pour des raisons évidentes, hétérodiégétique, et le second homodiégétique (idem).

S'ensuit, tout aussi notoirement, un affinement du second pôle, selon que le texte en exemplifie le degré fort (lorsque «le narrateur est le héros de son récit [Gil Blas]» [ibidem, p. 253]), baptisé "autodiégétique», ou le degré faible (lorsque le narrateur «ne joue [en tant que personnage] qu'un rôle secondaire, qui se trouve être, pour

10. Le chapitre s'achève en fait sur la prise en compte du narrataire. 
ainsi dire toujours, un rôle d'observateur et de témoin: [...] le Dr Watson de Conan Doyle» [idem]), qui ne fait pas l'objet d'une création néologique supplémentaire.

Or, en dépit de l'apparente clarté de cette mise au point typologique et terminologique, les définitions mêmes qui sont ainsi proposées ne vont pas sans autoriser certaines divergences interprétatives, qui expliquent assez largement les lectures biaisées qui seront ultérieurement faites du modèle théorique genettien. Passons provisoirement, avant d'y revenir par après, sur la présentation d'Homère et de Flaubert comme "narrateurs" respectifs de l'Iliade et de L'Education sentimentale ${ }^{11}$, qui, en l'absence de précision complémentaire, paraît tout de même contrevenir à la stricte dissociation antérieure des instances narrative et littéraire. Le nœud du problème réside ici, selon moi, dans l'absence de clarification de l'activité néologique. Autrement dit, les raisons qui président à l'élaboration du diptyque «homodiégétique» vs "hétérodiégétique» ne sont peut-être pas si "évidentes» que cela.

Certes, en dépit de flottements que Genette signalera lui-même rétrospectivement ${ }^{12}$, le "radical» "diégèse», sur lequel sont formés ces néologismes, dans la mesure où il est référé, non pas à la "diégésis» aristotélicienne, mais aux travaux d'analyse filmique d'Etienne Souriau ${ }^{13}$, qui distingue «univers diégétique» et "univers écranique», est compréhensible: il s'agit de l'univers spatio-temporel de l'histoire racontée, où évoluent, agissent et (le plus souvent) parlent les personnages. Certes, encore, les préfixes "hétéro-» et "homo-», en tant que tels, font sens; et, si ce n'était pas le cas, le Trésor de la langue française viendrait à point nommé rappeler aux lecteurs dans le besoin qu'il s'agit là de deux «éléments formants, issus du grec, [...] entrant dans la composition de très nombreux termes savants (substantifs et adjectifs)", dont le premier signifie "autre», et marque par là même "l'idée d'une différence de forme, de nature, de provenance, entre des individus, des espèces, des éléments"; quand le second signifie «semblable, le même», et veut donc dire l'inverse du précédent ${ }^{14}$.

Peut-être estimera-t-on, sur cette base, que je complique à plaisir une situation somme toute limpide, et me fais avec un peu trop de zèle l'avocat du diable (anti-genettien), puisque pour comprendre les termes en cause, il suffirait somme toute de biffer les mentions inutiles, c'est-à-dire inappropriées, dans les définitions qui précèdent? Mais encore faut-il choisir les bonnes, et l'évidence d'un tel choix est précisément contredite à longueur de volumes et d'articles. En d'autres termes, la question est ici celle de la nature de la relation d'altérité $v$ s d'identité (ou de similitude), ou, si l'on peut aller jusque-là, d'hétérogénéité $v s$ d'homogénéité, entre l'instance narrative et l'histoire qu'elle raconte. Est-elle d'ordre purement formel? Ontologique? Ou autre? Et/ou sur quel plan un narrateur peut-il être dit "étranger " à l'histoire qu'il relate? Sans doute le choix de formules plus strictement symétriques viendrait-il

11. Pour éviter de multiplier abusivement les notes bibliographiques, je ne référencerai que les textes littéraires non cités par Genette. Pour ceux qu'il évoque lui-même, on voudra bien se reporter à ses ouvrages et à leurs notes et/ou bibliographie.

12. Dans Nouveau Discours du récit, op. cit., p. 13-14.

13. Voir par exemple L'Univers filmique, Paris, Flammarion, 1953.

14. Entrées «Homo-» et "Hétéro-» du Trésor de la langue française informatisé (http://www.atil.atil. fr/), consultées le 2 avril 2015. 
dissiper certaines de ces zones d'ombre, en opposant un «narrateur présent comme personnage dans l'histoire qu'il raconte» (homodiégétique) et un "narrateur absent [comme personnage] de l'histoire qu'il raconte» (hétérodiégétique). L'adjonction est certes minime, et probablement sous-entendue dans la formulation originelle ${ }^{15}$; mais par le découplage fonctionnel (narrer $v$ s agir) qu'elle exhibe, peut-être contribuerait-elle à désamorcer certains risques de mécompréhension. Peut-être seulement, car je ne suis nullement persuadé que ce modeste réajustement suffise à prévenir tout danger de confusionnisme avec ce qui relève du niveau narratif - et qui découle assez largement de l'appréhension du narrateur en termes d'absence ou de présence dans la diégèse. Mais il est vrai que la responsabilité en incomberait non pas tant à Genette qu'à ses lecteurs ${ }^{16}$.

Toutefois, s'il présente comme évidentes les raisons qui président à ses choix néologiques, ce peut être aussi, tout bonnement, parce qu'il les avait déjà élucidées dans certains de ses travaux antérieurs. En effet, c'est dès 1968 qu'avait été introduite la distinction de l'homo- et de l'hétérodiégétique, à l'occasion d'une communication reprise l'année suivante dans Figures $I I$, sous le titre «D'un récit baroque ${ }^{17}$ ». Le double néologisme y avait été créé pour cerner la nature des relations entre des lignes d'histoire relevant de niveaux distincts - pour leur part baptisés «intra-» et "métadiégétique»-, lorsque surviennent des procédés d’enchâssement narratif, comme dans le Moïse sauvé de Saint-Amand:

Du point de vue du contenu narratif, [le] récit second peut être, par rapport au récit premier, soit homodiégétique, c'est-à-dire concernant par exemple les mêmes personnages que le récit principal (exemple, les récits d'Ulysse chez Alkinoos), soit hétérodiégétique, c'est-à-dire se rapportant à des personnages entièrement différents et donc en général à une histoire sans rapport de contiguïté avec l'histoire première [...]: exemple, le récit du Curieux impertinent dans Don Quichotte (p. 202).

Conformément à la valeur sémantique des préfixes choisis, la dialectique de l'identité et de l'altérité apparait ici avec une grande clarté; et l'hétérodiégétique peut être alors défini comme ce qui relève d'une autre ligne d'histoire. Cette acception du terme paraît d'ailleurs confirmée dans Palimpsestes (p. 343-344), où Genette propose de distinguer "transformations homodiégétiques" et "hétérodiégétiques ", à partir du critère de la conservation (travestissement) ou de l'altération (parodie) de l'identité des personnages et de leurs actions, lors du passage de l'hypotexte à l'hypertexte.

En tant que telles, ces définitions de l'homo- et de l'hétérodiégétique sont limpides, mais le problème, si problème il y a, provient de leur application à l'instance narrative primaire. Or, telle est bien l'acception la plus répandue de ces deux adjectifs, et celle

15. D'autant que, pour Genette, il est évident que ce n'est qu'en tant que personnage qu'un narrateur primaire peut être éventuellement présent dans l'histoire qu'il raconte, la question n'ayant pas de sens en termes de niveaux, puisque, considéré sous l'angle de cet autre paramètre, le narrateur primaire est par définition extradiégétique. Que le principal intéressé daigne donc pardonner mes divers "points", à ses yeux sans doute superflus, voire absurdes, sur ces «i» narratologiques. Mais nécessité fait loi...

16. Voir la note précédente.

17. P. 195 à 222 de l'édition citée. 
que leur donne ultérieurement Genette dans Nouveau Discours du récit (p. 63-73), puis dans sa correspondance avec Dorrit Cohn, publiée sous le titre de "Nouveaux nouveaux discours du récit ${ }^{18}$ ». Pour cerner les difficultés qui surgissent alors, il est donc indispensable (et plus que temps) de présenter, même sommairement, la typologie genettienne du niveau narratif.

A déjà été mentionnée incidemment la distinction de l'intra- et du métadiégétique, opérée dans Figures II pour présenter les deux types d'amplification par expansion que recèle Moïse sauvé. Mais c'est de Figures III que date la formalisation d'ensemble du phénomène, fondée sur le principe que "tout événement raconté par un récit est à un niveau diégétique immédiatement supérieur à celui où se situe l'acte narratif producteur de ce récit» (p. 238); d'où la triade "extradiégétique» (de premier niveau $=$ extérieur à la diégèse)/intradiégétique (de deuxième niveau = intérieur à la diégèse)/métadiégétique (de troisième niveau = interne à une diégèse elle-même comprise dans une autre diégèse). Peut-être cette conception "architecturale» du récit fictionnel est-elle potentiellement fourvoyante, en raison du caractère contre-intuitif de la logique ascensionnelle qui la sous-tend ${ }^{19}$; et l'on peut estimer qu'une schématisation par imbrication de phylactères rendrait mieux compte des phénomènes d'enchâssement narratif: ce sera chose faite dans Nouveau Discours du récit (p. 56-57). Toutefois, quel que soit le type de schéma proposé, l'essentiel est de présenter l'activité narrative elle-même comme une frontière délimitant divers niveaux; mais qui, comme toute frontière, peut être transgressée - par voie de métalepse. Dont acte.

Quant à la détermination des "quatre types fondamentaux du statut du narrateur" (extradiégétique-hétérodiégétique/extradiégétique-homodiégétique/intradiégétique-hétérodiégétique/intradiégétique-homodiégétique - Figures III, p. 255 sq.), loin d'autoriser une confusion de la relation de personne et du niveau, elle devrait au contraire favoriser leur distinction, puisque l'articulation de ces paramètres suppose au préalable que leur dualité soit perçue. Or, ce modèle, qui a pourtant le mérite de la clarté, a suscité de très nombreuses erreurs d'interprétation, au point d'inciter Genette à se fendre d'une note explicative, dont on goûtera (ou non...) l'ironie:

Le double statut de Renoncour (et de bien d'autres de même type) semble parfois mal compris; c'est pourtant simple: il est intradiégétique comme narrataire de Des Grieux et extradiégétique comme narrateur (auteur fictif) du récit primaire de Manon Lescaut. Et il ne peut être les deux que parce qu'il est narrateur homodiégétique, c'est-à-dire présent comme personnage (entre autres, comme narrataire)

18. Poétique, n 61, février 1985, p. 101-109, rubrique "Débat".

19. Sur ce point, voir Pierre Bayard, Aurais-je sauvé Geneviève Dixmer?, Paris, éd. de Minuit, «Paradoxe», 2015, p. 25: «Cette différence de niveau apparaît d'ailleurs dans les métaphores qui viennent naturellement à l'esprit quand on essaie de se figurer l'entrée dans un livre [...]. L'image qui s'impose naturellement pour un livre est que l'on y descend." Il s'agit certes là d'une réflexion sur la métalepse, mais Bayard n’y présente pas moins clairement, à l'inverse de Genette, le niveau intradiégétique comme situé au-dessous du niveau extradiégétique. Chacun jugera par lui-même de la dimension plus ou moins «naturelle» de ces logiques spatialement contradictoires. 
dans le récit qu'il assume. Tout narrateur extra-homodiégétique est intradiégétique comme personnage et extradiégétique comme narrateur. Vu? (Nouveau Discours $d u$ récit, p. 91, note 1 ).

Vu... Pour s'y retrouver, il n'est que de se souvenir que ces termes (extra-, intra-, métadiégétique) «désignent non pas des êtres, mais des situations relatives et des fonctions» (Figures III, p. 239), ce qu'illustre en effet parfaitement le narrateur primaire homodiégétique, qui, dans la perspective genettienne, exerce son activité narrative (raconter qu'il s'est longtemps couché de bonne heure) depuis l'extérieur de la diégèse, alors qu'il ne peut agir (se coucher longtemps de bonne heure), ès qualités de personnage, qu'à l'intérieur de celle-ci.

De fait, ce n'est pas à propos de ce type narratif que se posent les questions théoriques les plus stimulantes ${ }^{20}$. Tout juste pourrait-on formuler deux observations incidentes : tout d'abord, au sein même de la relation de personne homodiégétique, il ne paraît pas toujours aisé d'évaluer le degré (fort ou faible) en cause. En particulier, dans les romans contemporains, où le système des personnages se trouve notoirement déhiérarchisé, il est ainsi souvent malaisé de trancher entre relations auto- ou homodiégétique. Etablir avec assurance le seuil à partir duquel il serait légitime d'affirmer qu'un narrateur-personnage raconte son histoire, c'est-à-dire y occupe le premier rôle actantiel, ne paraît donc pas aller de soi - et, à vrai dire, de moins en moins au fil de l'évolution des formes romanesques. Mais sans doute n'est-ce là qu'un demi-problème (et encore...), puisque seul le degré de la relation de personne est alors concerné. Ensuite, peut-être aura-t-on trouvé surprenant que Genette classe les récits dont la narration est conduite à la deuxième personne (en "vous»: La Modification de Butor; en «tu»: Un homme qui dort de Perec) au sein du type hétérodiégétique ${ }^{21}$. Certes, eu égard à ses propres définitions, cette classification est parfaitement légitime, puisque, alors, ce n'est pas le narrateur mais le narrataire qui est présent comme personnage de l'histoire. Mais, en vertu de la symétrie inhérente à une conception communicationnelle du récit, ne pourrait-on à l'inverse estimer que la narration "à la deuxième personne» constitue plutôt une variante de la relation homodiégétique, en ce qu'elle en est en définitive plus proche structurellement que de la relation hétérodiégétique? Définir la narration comme "l'acte producteur et, par extension, l'ensemble de la situation réelle ou fictive dans laquelle il prend place» (Figures III, p. 72) pourrait, je crois, y inciter - sans qu'il y ait là matière à controverse.

Bref, sauf à confondre les paramètres du niveau et de la personne, à l'examen, la relation homodiégétique telle que la définit Genette ne paraît guère susciter de problèmes théoriques majeurs ${ }^{22}$, et n'a de fait, à ma connaissance, pas fait l'objet

20. A l'exception notable des positions défendues par Käte Hamburger dans Logique des genres littéraires (1957 puis 1968; traduction anglaise en 1973), Paris, éd. du Seuil, «Poétique», 1986, pour la traduction française, préfacée par Gérard Genette. L'auteur y exclut les récits «à la première personne» (homodiégétiques, dans la terminologie genettienne) du champ de la fiction.

21. Dans Nouveau Discours du récit, op. cit., p. 92.

22. De nouveau, exception faite de la position de Käte Hamburger, évoquée dans la note 20. Pour une 
de désaccords notables de la part des autres narratologues. Cet apparent consensus peut s'expliquer par le caractère en l'occurrence indiscutable de la dissociation de l'auteur et du narrateur primaire; puisque nul théoricien du récit ne saurait confondre Albert Camus et Meursault ${ }^{23}$, la Comtesse de Ségur et Cadichon ${ }^{24}$, ou encore Mark Twain et un bacille du Choléra ${ }^{25}$... En revanche, il en va tout autrement de la relation hétérodiégétique, à propos de laquelle se font jour des opinions pour le moins contrastées, voire diamétralement opposées; de sorte qu'il est nécessaire à présent de s'y attarder.

\section{Problématologie: de quelques points borgnes, sinon aveugles}

C'est en effet le cas particulier de la narration "à la troisième personne» ("hétérodiégétique», dans la terminologie genettienne) qui a conduit certains théoriciens, hostiles à la théorie communicationnelle du récit, à considérer le narrateur comme une instance "optionnelle». Chacun se souvient sans doute de la polémique ayant opposé Gérard Genette et Ann Banfield à ce sujet ${ }^{26}$, qu'a ravivée la parution de l'ouvrage de Sylvie Patron, Le Narrateur. Entrer dans le détail de cette controverse excéderait très largement le cadre nécessairement restreint de mon propos; aussi me contenterai-je de signaler que, même si les arguments linguistiques des tenants de l'hypothèse du récit sans narrateur ne me paraissent guère convaincants, pour les raisons que Florian Pennanech a très clairement exposées ${ }^{27}$, il n'en est pas moins compréhensible que le narrateur extra-hétérodiégétique passe à certains égards pour une instance problématique. Sont en effet plus particulièrement susceptibles de poser question(s) sa relation à l'instance littéraire, son degré d'individuation, son rapport

présentation détaillée de sa théorie, voir Sylvie Patron, Le Narrateur. Introduction à la théorie narrative, Paris, Armand Colin, «U», 2009, chap. 7 («Pour une autre lecture de Hamburger»), p. 151-176.

23. Albert Camus, L'Etranger, Paris, Gallimard, 1942.

24. Sophie Rostopchine, Comtesse de Ségur, Les Mémoires d'un âne, Paris, Hachette, 1860. Que, du traitement de cet exemple, on n'infère de ma part nulle conviction "spéciste». Quant à savoir, plus généralement, si l'animalitarisme est ou non un antihumanisme, je laisse à d'autres, mieux "équipés" que moi pour ce faire, cet os à ronger...

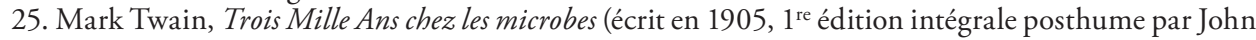
Tuckey, en 1980, dans The Devil's Race-Track), Paris, éd. de la Différence, 1985 pour la traduction française.

26. La position d'Ann Banfield est exposée dans Phrases sans parole. Théorie du récit et du style indirect libre (1982), Paris, éd. du Seuil, «Poétique», 1995, pour la traduction française; celle de Gérard Genette aux pages 66 à 69 de Nouveau Discours du récit, op. cit.

27. Florian Pennanech, «La mort du narrateur (ou pas)», Acta fabula, vol. 10, n 10, Essais critiques, décembre 2009, URL: http://www.fabula.org/acta document5332.php

Page consultée le 2 avril 2015. Voir en particulier la page 7, où Florian Pennanech dénonce le manque de pertinence des arguments linguistiques (disons, très schématiquement, la présence de passages en style indirect libre comme "preuve" de l'inexistence de l'instance narrative; voir Le Narrateur, op. cit., en particulier les chapitres 8 et 9) des tenants du récit sans narrateur, en mettant en exergue le cas du narrateur intra-hétérodiégétique (exemple: Schéhérazade), dont il paraît en effet pour le moins difficile de contester l'existence. 
à l'univers diégétique, ou encore son «mode d'être» ou, si l'on préfère, son statut ontologique - questions qu'il n'est d'ailleurs pas toujours aisé de clairement distinguer.

Tout d'abord, et contrairement à un truisme critique dont on sait ce qu'il doit aux recherches linguistiques d'Emile Benveniste ${ }^{28}$, selon moi, la question de la détermination de la source de la parole narrative, pour être moins cruciale qu'en relation homodiégétique, n'en conserve pas moins une importance certaine en relation hétérodiégétique - c'est là, je crois, l'un des enseignements possibles de "Frontières du récit». Mais, à partir du moment où elle ne se matérialise jamais en tant que personnage de l'histoire racontée, comment caractériser cette instance qui émaille le texte proposé aux lecteurs de sèmes évaluatifs et autres indices de subjectivité? Un réflexe somme toute compréhensible serait alors de faire l'économie du narrateur, et d'estimer que la voix qui résonne ainsi dans l'espace du texte, puis dans l'esprit de ceux qui le lisent, émanerait tout bonnement et directement de l'auteur - position qu'encouragent d'ailleurs certains écrivains, par exemple Milan Kundera ${ }^{29}$.

Le problème, si c'en est un ${ }^{30}$, tient à ce que seraient ainsi sapés les fondements mêmes de l'édifice narratologique, qui semble reposer, comme on l'a vu, sur la distinction apriorique de l'auteur et du narrateur. Mais est-ce réellement le cas? Et à quel degré? Certaines affirmations genettiennes paraissent certes corroborer et motiver la nécessité de ce distinguo, y compris en relation hétérodiégétique, comme l'atteste cet important passage de Figures III:

[...] le narrateur du Père Goriot n'«est» pas Balzac, même s'il exprime çà ou là les opinions de celui-ci, car ce narrateur-auteur est quelqu'un qui «connâ̂t» la pension Vauquer, sa tenancière et ses pensionnaires, alors que Balzac, lui, ne fait que les imaginer (p. 226).

Mais on constate qu'une certaine ambiguïté se fait alors jour. Ainsi, si la distinction des instances est affirmée, elle n'en est pas moins quelque peu relativisée, d'une part par la concessive («même si...»), d'autre part par l'emploi de guillemets de

28. «De la subjectivité dans le langage» (1958), et «Les relations de temps dans le verbe français» (1959), dans Problèmes de linguistique générale, vol. I, Paris, Gallimard, 1966, puis «Tel», 1990, pour l'édition utilisée. Les articles en cause s'y trouvent respectivement aux pages 258-266 et 237-250. On se souvient de la définition suivante de l'objectivité présumée du récit (par opposition à la subjectivité du discours) : «A vrai dire, il n’y a même plus de narrateur. Les événements sont posés comme ils se sont produits à mesure qu'ils apparaissent à l'horizon de l'histoire. Personne ne parle ici; les événements semblent se raconter eux-mêmes» (op. cit., p. 241). Pour une relecture narratologique de la position de Benveniste, on se reportera à l'article de Genette, «Frontières du récit» (Figures II, op. cit., p. 49-69).

29. Par exemple dans "Questions et réponses échangées par écrit entre André-Alain Morello et Milan Kundera", Dix-neuf/Vingt, n 1, mars 1996 (cité par Sylvie Patron, Le Narrateur, op. cit., p. 301-302). Ce qui, pour autant, ne "prouve» rien, d'une part parce qu'il ne manque pas d'écrivains pour défendre, à l'inverse, l'hypothèse de l'existence du narrateur (Robbe-Grillet, entre autres), d'autre part parce que les sphères de compétence des romanciers et des narratologues n'ont aucune raison de se superposer, sans reste. Autrement dit, un théoricien du récit n'a pas à se considérer comme esclave des opinions des auteurs sur leur propre pratique d'écriture pour ce qui a trait à l'élaboration de ses propres outils d'analyse. Que les uns et les autres ne se posent pas nécessairement les mêmes questions ni n'y apportent les mêmes réponses paraît relever de l'évidence.

30. Ce devrait tout de même en être un pour les narratologues... 
précaution. Peut-être y a-t-il là, de ma part, risque de sur- ou de mésinterprétation, mais la modalisation du verbe "être" peut, je crois, se lire à la fois comme l'indice de la complexité de la relation qui unit ici le narrateur du Père Goriot et Balzac (ni tout à fait le même, ni tout à fait un autre), et comme l'indice du statut paradoxal de cette instance (qui ne possède pas d'existence, au sens usuel du terme). De même, les guillemets environnant le verbe "connaître» peuvent désigner simultanément le statut paradoxal du narrateur extra-hétérodiégétique et de l'«objet» (la pension Vauquer, dépourvue d'existence en dehors des pages du roman de Balzac) de sa "connaissance». On regretterait presque que le pronom indéfini "quelqu'un" n'ait pas fait l'objet d'une modalisation similaire, tant le mode d'être de l'instance qu'il désigne semble spécifique - mais il est vrai que, stylistiquement, point trop n'en faut... En outre, pour tenter de clarifier plus avant la situation, peut-être pourrait-on préférer le verbe épistémique "croire à» au verbe "connaître» choisi par Genette. En effet, en dépit de leurs similitudes, telle est bien en l'occurrence la différence majeure entre instances littéraire et narrative: l'une (Balzac) ne croit pas à l'existence de la pension Vauquer et de ses habitants (puisque elle les invente); l'autre (le narrateur du Père Goriot) y croit, et témoigne ainsi d'un rapport de phase à l'univers diégétique qu'elle évoque, quand bien même elle ne sý manifeste jamais en tant que personnage, se contentant de décrire et de narrer : à chacun selon ses moyens. Un tel réajustement permet de préciser que ce qui sépare l'auteur du narrateur relève des relations entre «domaines d'existence ${ }^{31}$ »: entre l'auteur et la diégèse, il y a non-identité desdits domaines; entre le narrateur et la diégèse, identité - ce que confirme l'affirmation genettienne selon laquelle "le récit de fiction n'est qu'une fiction de récit ${ }^{32}$ », que Florian Pennanech traduit pour sa part par «relation fictive de faits ${ }^{33}$ ». Il est donc possible d'en inférer que l'instance qui assume une telle relation est elle-même fictive ("fictionnelle» conviendrait mieux). C'est en ce sens que l'on peut comprendre que "le narrateur auctorial [puisse] être à la fois fictionnel et hors fiction (situé à l'extérieur du monde fictionnel des personnages) ${ }^{34}$ ", puisque la question de son statut ontologique ne saurait se résumer à celle du niveau auquel il émarge. Si cette "question cruciale" (idem) paraît parfois insoluble, c'est précisément parce que "hétérodiégétique» est indûment traduit par «externe», et «homodiégétique» par «interne $35^{35}$,

31. Notion présente chez Franz K. Stanzel ( $A$ Theory of Narrative [1979], Cambridge, Cambridge University Press, 1984, pour la traduction anglaise), mais qui n'en fait pas le même usage. Sur ce point, voir Le Narrateur, op. cit., p. 83, 93 et passim.

32. Dans la préface déjà mentionnée de Logique des genres littéraires (op. cit.), reprise, sous le titre de "Une logique de la littérature», dans Figures IV, Paris, éd. du Seuil, «Poétique», 1999, p. 331.

33. «La mort du narrateur (ou pas)», art. cité, p. 2. J'ajouterais toutefois, pour ma part, que la dimension fictionnelle du «contenu» narratif mériterait elle aussi d'être prise en considération. "Relation fictive de faits fictionnels» est sans doute "un peu» lourd (et/car redondant)? Ma foi, tant pis, puisque c'est bien de cela qu'il s'agit.

34. Le Narrateur, op. cit., p. 93.

35. Par exemple chez/par Seymour Chatman, Story and Discourse: Narrative Structure in Fiction and Film, Ithaca et Londres, Cornell University Press, 1978; comme chez/par Sylvie Patron, Le Narrateur, op. cit., p. 63. Il y a là confusion manifeste de ce qui relève de la personne (homodiégétique ou hétérodiégétique) et du niveau (extradiégétique ou intradiégétique), de sorte que ce qui se trouve, sur cette base, en cause, n'est pas tant le modèle élaboré par Genette que la lecture biaisée qui en est faite à partir d'une compréhension partielle. «Interne» et « externe» sont abusivement simplificateurs, mais tout lecteur attentif 
ce qui revient à fausser les données du problème, en écrasant maladroitement un paramètre sous l'autre.

Pour autant, cette mise au point est bien loin de résoudre toutes les difficultés. Ainsi, même les tenants de la narratologie genettienne peuvent éprouver quelque gêne face à une telle anthropomorphisation ("quelqu'un qui...») de ce qui n'est en toute rigueur qu'une instance textuelle. Mais, après tout, si l'on convient que le langage articulé est le propre de l'homme ${ }^{36}$, peut-être n'est-il pas si embarrassant d'appréhender en ces termes la source de la parole narrative. D'autant que, quelle que soit la relation de personne à l'œuvre, le narrateur peut être crédité d'un ethos $^{37}$, c'est-à-dire que les modalités de son dire esquissent une image de cette instance, à charge pour les critiques, et plus généralement pour les lecteurs, d'en cerner les contours. Si l'on me passe le "perecquisme» qui suit, la formule "ontologique» du narrateur pourrait donc être la suivante: "pour être, besoin d'éthè 38 "; à condition de préciser que ce besoin est toujours satisfait, même dans le cas des narrations les plus neutres ou "objectives» (relevant du «showing»), qui connotent alors l'impassibilité de l'instance source - l'activité interprétative, on le sait, ne chôme jamais...

Mais les difficultés les plus épineuses surviennent lorsque certaines des affirmations de Genette lui-même paraissent contrevenir au distinguo auteur vs narrateur. A déjà été mentionnée la présentation ponctuelle d'Homère ou de Flaubert comme narrateurs respectifs de l'Iliade et de L'Education sentimentale: j'ignore s'il faut y voir un simple retour formulaire du refoulé "prénarratologique», comme tel dénué de réelles implications épistémologiques. Quoi qu'il en soit, tel n'est à l'évidence pas le cas dans cet extrait de Nouveau Discours $d u$ récit, qui, si on le confronte au passage précédemment glosé de Figures III, risque de vouer les plus genettiens des «genettiens» à la perplexité:

Il est absolument légitime de distinguer en principe le narrataire du lecteur, mais il faut aussi tenir compte des cas de syncrèse. De même, bien sûr, le narrateur extradiégétique se confond totalement avec l'auteur - je ne dirais pas, comme on le fait trop, «implicite», mais bel et bien explicite et proclamé. Je ne dis pas non plus « réel », mais tantôt (rarement) réel, comme, disons, le Giono de Noé, reconnaissable à sa robe de chambre «taillée dans une couverture de cheval toute rouge», et autres détails autobiographiques; tantôt fictif (Robinson Crusoé) ; tantôt quelque bizarre hybride des deux, comme l'auteur-narrateur de Tom Jones, qui n'«est» pas Fielding, mais n'en pleure pas moins, une ou deux fois, sa défunte Charlotte (p. 91-92).

de Genette devrait percevoir que le premier des deux adjectifs ne saurait s'appliquer qu'au narrateur intradiégétique, le second au narrateur extradiégétique.

36. Je laisse donc de côté le cas des mainates et autres oiseaux parleurs. Sur les présupposés qui sous-tendent cette «exclusion», on voudra bien se reporter à la note 24 .

37. Sur cette notion, voir Ruth Amossy, «La double nature de l'image de l'auteur», Argumentation et analyse $d u$ discours [en ligne], 3, 2009, URL: http://aad.revues.org/662

Article consulté le 2 avril 2015.

38. Georges Perec, Wou le souvenir d'enfance, Paris, Denoël, 1975, p. 77 : «Pour être, besoin d'étai.» Dans ma "tra(ns)duction", c'est, on l'aura compris, pour des raisons d'homophonie que s'impose le pluriel d'ethos. 
Si le cas de Robinson Crusoé ne pose aucun problème, si celui du narrateur de Tom Jones est déjà beaucoup plus embarrassant, celui du Giono de Noé vient en apparence remettre en question les principes mêmes de la définition narratologique de la voix narrative. Voilà en tout cas qui confirme, aux yeux de Genette, la prévalence des manifestations textuelles empiriques sur les cadres taxinomiques des théoriciens, comme, à rebours de la réputation qui lui a trop hâtivement été prêtée, sa propension à tempérer l'immanence de l'approche narratologique d'un zeste de transcendance ${ }^{39}$. Sans doute est-ce tout à l'honneur du poéticien, mais la question est alors de savoir si le «cas de syncrèse» exceptionnel exemplifié par Noé confirme ou, à l'inverse, infirme la règle - et la réponse n'a rien d'évident.

Toutefois, même s'il ne s'agit pas d'un hapax, force est de convenir de l'extrême singularité de l'exemple, puisque Giono s'y met en scène à sa table de travail, sur laquelle déambulent les personnages de son roman précédent, Un roi sans divertissement. Une telle combinaison de métalepse et de transfictionnalité ${ }^{40}$ est donc bien loin de représenter le régime courant de la fiction narrative. En outre, on aura noté que Genette n'identifie pas là une substitution de l'auteur réel au narrateur, mais un cas de confusion des deux instances, dont la relation "symbiotique" ne suffit pas à conclure à l'éradication de la seconde au bénéfice de la première. Mais, aussi particulier soit-il, ce cas rare n'en incite pas moins à poser un certain nombre de problèmes cruciaux, tels que le degré d'individuation du narrateur, la diversité de ses types possibles, sa faculté à faire intrusion dans le récit (je dis bien «dans le récit ${ }^{41}$ ), qui demeurent pertinents à propos de textes moins anomiques. La difficulté majeure tient en l'occurrence à l'effet de signature inhérent à la mention de « détails autobiographiques", dont on a vu qu'il valait également, à un degré moindre, pour le narrateur de Tom Jones. Qu'en déduit Genette, dans le cas de ce second exemple? «Le narrateur est donc Fielding, mais feignant en partie une personnalité qui n'est pas la sienne ${ }^{42}$ » (Nouveau Discours du récit, p. 100, note 1). Largument pourrait de prime abord paraître trop ingénieux pour être honnête, car fondé sur une apparente tautologie ${ }^{43}$, mais il me semble rendre compte avec justesse de la complexité de la scénographie narrative en régime fictionnel. Si l'auteur ne peut assurer directement (sans intermédiaire) l'activité narrative, c'est en raison de la non-identité des domaines

39. Ce que confirme amplement l'un de ses articles, «Peut-on parler d'une critique immanente?», Poétique, 126, avril 2001, p. 131-150.

40. Richard Saint-Gelais, Fictions transfuges. La transfictionnalité et ses enjeux, Paris, éd. du Seuil, «Poétique», 2011.

41. Je reconduis ici la distinction «narration»/«récit»/«histoire» posée dans Figures III, op. cit., p. 72.

42. On observera en passant que cette idée rejoint en apparence celle de «second moi» qui, selon Wayne Clayton Booth (The Rhetoric of Fiction, Chicago, Chicago University Press, 1983), permet de définir l'auteur "impliqué» (ou «implicite», selon les traductions de la formule "implied author»). A cette importante différence près que, chez Booth, l'«implied author» ne peut pas intervenir dans son œuvre en tant que sujet énonciateur. En outre, Genette considère l'auteur impliqué comme une instance inutile (voir Nouveau Discours du récit, op. cit., p. 96 sq.); opinion dont on pourrait tout de même débattre, notamment dans le cas des narrateurs primaires ironisés. Mais ce sera(it) là l'objet d'une autre étude, intitulée "Quand le narrateur boit(e)... (Réflexions sur le narrateur non fiable et/ou indigne de confiance) ", à paraitre dans la revue en ligne COnTEXTES...

43. Puisque tout récit fictionnel suppose un narrateur, même lorsqu'il s'agit de l'auteur, une différence (ici, «de personnalité») doit bien exister, qui permette de sauver l'hypothèse du narrateur. 
d'existence déjà évoquée, et de ses implications en termes de "croyance » à l'univers de la diégèse. Le narrateur de Tom Jones a beau pleurer «sa» défunte Charlotte, il n'en croit pas moins à l'existence de l'univers spatio-temporel où évolue le héros éponyme, ce qui devrait suffire à le distinguer de Henry Fielding.

Les problèmes indéniables qui se posent malgré tout tiennent à l'individuation de l'instance narrative sous les aspects d'un «narrateur-auteur» ou d'un «narrateur auctorial»; ce qui pourrait à première vue paraître relever d'une altération de la pureté typologique: la caractérisation du narrateur primaire ne contribuerait-elle pas ipso facto à l'éloigner du type hétérodiégétique? A l'examen, pas nécessairement, d'une part parce que l'individuation de l'instance narrative est alors une conséquence du déploiement de sa propre parole, d'autre part parce que l'autoportrait en creux qui s'esquisse ainsi ne suffit pas à en faire un personnage de la diégèse. Même les moins discrets des narrateurs primaires, ou, si l'on préfère, les plus «intrusifs» d'entre eux, n'en deviennent pas pour autant des entités intradiégétiques. Pour l'établir, il importe donc d'évoquer conjointement les questions du choix pronominal et de l'intervention narratoriale. $\mathrm{Si}$ «La vraie question est de savoir si le narrateur a ou non l'occasion d'employer la première personne pour désigner l'un de ses personnages» (Figures III, p. 252), il s'ensuit que de la relation hétérodiégétique relèvent également les récits où l'emploi de la première personne du singulier ou du pluriel correspond à «la désignation du narrateur en tant que tel par lui-même, comme lorsque Virgile écrit "Arma virumque cano..." (idem). Aussi contre-intuitive cette idée puisse-t-elle paraitre, une narration conduite «à la première personne» (au sens grammatical du terme: choix du pronom «je» ou «nous») peut donc constituer une variante de la narration hétérodiégétique, aux conditions de «référence» qui viennent d'être précisées.

Toutefois, la plupart du temps, en régime d'hétérodiégéticité, c'est seulement ponctuellement que l'impersonnalité de convention est congédiée, à l'occasion de l'apparition impromptue d'un pronom de la première personne du singulier ou du pluriel. Que l'on pense par exemple au récit balzacien, où un "nous» des plus envahissants se manifeste à intervalles réguliers pour légitimer des choix narratifs auxquels les lecteurs du XIX ${ }^{\mathrm{e}}$ siècle n'étaient pas encore accoutumés ${ }^{44}$. Est ainsi spectaculairement confirmée l'idée que «tout récit est, explicitement ou non, "à la première personne", puisque son narrateur peut à tout moment se désigner par ledit pronom» (Nouveau Discours du récit, p. 65). Bien sûr, de la multiplicité de ces «excursus» résulte une forme de caractérisation de l'instance qui les assume ${ }^{45}$; mais, une fois encore, les pronoms «nous» et/ou «je» renvoient alors à l'instance narrative primaire en tant que telle, de sorte que le portrait (en fait l'autoportrait) qui apparaît en filigrane de ces «interventions» récurrentes n'a pas à être considéré comme celui d'un personnage de la diégèse. Quelle que soit sa fréquence, un tel procédé n'invalide donc pas l'appartenance des récits où il apparait au type hétérodiégétique.

44. Ce qui apparaît clairement à la lecture de l'article de Genette, "Vraisemblance et motivation », dans Figures II, op. cit., p. 71-99.

45. Ce qui paraît en effet altérer la pureté typologique, en raison de la remise en cause qui s'ensuit de la "neutralité», voire de «l'objectivité» communément associées à la relation hétérodiégétique - mais que les narrations relevant du "telling» fragilisent déjà notablement, sans cesser pour autant de relever de cette relation de personne. 
Pour mieux le comprendre, il peut être utile d'insister sur le caractère potentiellement trompeur de la dénomination d' «intrusion d'auteur ${ }^{46}$ ", comme, à un degré moindre, de sa reformulation narratologique en «intrusion du narrateur». En effet, en toute rigueur, il n'y a pas là de réelle intrusion, au sens où le narrateur primaire (par essence extradiégétique) pénétrerait à l'intérieur de la diégèse, et y évoluerait conséquemment avec un statut identique à celui des personnages qui la peuplent. Certes, les conséquences dénudantes du procédé sont indéniables, qu’elles soient recherchées (dans Jacques le fataliste de Diderot) ou subies (dans La Comédie humaine de Balzac), et ce notamment en raison de leurs implications temporelles; mais il n'y a là somme toute qu'une variante rhétorique de la métalepse, qu'il importe de distinguer de ses variantes ontologiques ${ }^{47}$. En tout état de cause, eu égard aux définitions genettiennes, cette autodésignation d'un narrateur extra-hétérodiégétique par l'emploi d'un pronom personnel n'entraîne donc pas d'altération de la relation de personne.

En revanche, cela ne signifie pas que ladite relation soit toujours invariable à l'échelle d'un récit, ni que la frontière entre les deux types (homo- $v$ s hétéro-) soit toujours évidente à déterminer. Ainsi, entre autres exemples, Genette analyse-t-il Madame Bovary, où la relation de personne évolue notoirement de l'homodiégétique à l'hétérodiégétique (le narrateur-témoin initial évacuant sans crier gare la scène du récit et de l'histoire), et identifie-t-il comme une «transgression plus forte encore [...] le changement de personne grammaticale pour désigner le même personnage» (Figures III, p. 253) ; phénomène dont «La forme de l'épée» de Borges vient fournir le paradigme.

Sur le plan théorique, plus que l'incontestable mobilité de la relation de personne sur la diachronie de certains récits, c'est la ténuité, voire la porosité de la frontière censée séparer ses deux types constitutifs, qui mérite de retenir l'attention. Et, dans cette perspective, la relecture du roman de Flaubert à laquelle se livre Genette dans Nouveau Discours du récitse révèle particulièrement éclairante: l'usage du présent dans les dernières lignes du roman (notamment dans la célébrissime clausule: «Il vient de recevoir la Croix d'honneur») introduirait selon lui «une dose [...] d'homodiégéticité dans le récit» (p. 53) - ce qui, soit dit en passant, démontrera à qui en douterait à quel point l'activité formalisatrice genettienne relève d'une poétique ouverte ${ }^{48} \ldots$ A fortiori, ce diagnostic peut valoir pour une narration hétérodiégétique intégralement conduite au présent, puisque ce temps «suggère presque irrésistiblement une présence du narrateur [comprendre: comme personnage] dans la diégèse" (Nouveau Discours du récit, p. 55). Les exemples choisis par Genette (Les Gommes, La Jalousie, Le Vice-consul) viennent en effet corroborer l'hypothèse d'un «effet [...] d'homodiégétisation" (idem) lié à l'emploi narratif du présent de l'indicatif.

Plus que le fait grammatical en tant que tel, ce qui importe dans le cas de la

46. Que Genette réfère à Georges Blin, Stendhal et les problèmes du roman, Paris, Corti, 1954.

47. Sur la distinction entre ces types de métalepse, voir John Pier et Jean-Marie Schaeffer (dir.), Métalepses. Entorses au pacte de la représentation, Paris, éd. de l'EHESS, «Recherches d'histoire et de sciences sociales", $\mathrm{n}^{\circ} 8,2005$.

48. Sur ce point, voir Christine Montalbetti, Gérard Genette. Une poétique ouverte, Paris, BertrandLacoste, 1998. 
narration hétérodiégétique au présent est donc la relation de contemporanéité (entre le narrateur et les faits évoqués dans la diégèse) qu'il est possible d'en inférer ${ }^{49}$; ce qui nous renvoie par un autre biais à la question de l'identité des domaines d'existence. De ce rapport de contiguïté relèveraient donc le «narrateur-contemporainconcitoyen", le "chroniqueur» (ibidem, p. 70-71), etc., qui paraissent bien témoigner de «l'existence [...] de situations frontalières, mixtes ou ambiguës» (idem, p. 71) en matière de relation de personne. Aussi Genette est-il fort logiquement conduit à relativiser «l'idée d'une frontière infranchissable entre [les] deux types hétéro- et homodiégétique» (idem, p. 70), et ce en faveur de la "possibilité d'une gradation progressive» (idem), rejoignant pour partie les hypothèses de Franz K. Stanzel ${ }^{50}$.

On le voit, si Genette élabore une typologie rigoureuse, en refusant de confondre choix pronominaux et attitudes narratives, niveaux et relations de personne, sa prise en compte de la diversité des productions textuelles et son ouverture à l'anomie lui permettent d'éviter l'écueil de la rigidité taxinomique ${ }^{51}$. En conséquence, et pour ce qui nous concerne, cette conception en définitive plus souple qu'il n'y paraît des relations de l'instance narrative et de la diégèse du récit fictionnel peut «rencontrer » certaines des mutations les plus originales du traitement de ce paramètre par les écrivains contemporains ${ }^{52}$.

\section{Tératologie: du côté de chez quelques écrivains contemporains ${ }^{53}$}

En effet, dans la littérature narrative du second $\mathrm{xx}^{\mathrm{e}}$ siècle comme du début du $\mathrm{xxI}^{\mathrm{e}}$ siècle, les exemples d'un traitement déceptif, et comme tel troublant, de la relation de personne sont légion. Par exemple, si Genette mentionne Le Vice-Consul de Marguerite Duras, Le Ravissement de Lol V. Stein ${ }^{54}$ du même auteur constitue en la matière un cas d'école. Il est d'ailleurs saisissant de constater à quel point l'étude de la voix narrative dans ce récit a pu donner lieu à des diagnostics erronés; mais sans doute la prise en compte d'un tel paramètre, vulgairement narratologique, ne

49. Du moins dans les cas qui viennent d'être évoqués, puisqu'on sait que le présent de l'indicatif peut posséder d'autres valeurs.

50. Telles qu'exposées dans Narrative Situations in the Novel, Tom Jones, Moby-Dick, The Ambassadors, Ulysses (1955), Bloomington et Londres, Indiana University Press, 1971, pour la traduction anglaise.

51. Conformément au principe qu'il énonce à la page 19 de Nouveau Discours du récit (op. cit.): «La rigidité est la rigueur des cuistres, qui ne sauraient jamais rien négliger.» De fait, en matière de formalisation théorique, comme ailleurs, certains «excès de zèle» évoqueraient plutôt la rigor mortis...

52. Sans que cela n'entraîne une rigoureuse superposition des domaines d'activité du poéticien et des écrivains: voir la note 29.

53. La place me fait défaut pour justifier la "contemporanéité» d'écrivains défunts comme Duras ou Robbe-Grillet. Et, à vrai dire, non seulement la place, mais l'envie: disons que je désigne simplement, par défaut, comme "contemporains» divers auteurs dont la production court des années 1960 à nos jours.

54. Paris, Gallimard, 1964. 
représentait-elle qu'une concession de pure forme (sic) aux «exigences» de l'air du temps ${ }^{55}$, de la part de critiques impatients de se lancer à corps perdu dans l'exploration vertigineuse des abîmes de l'être. Pour autant, la plus élémentaire maîtrise des règles d'accord du participe passé invalide d'emblée, et ce dès l'incipit ${ }^{56}$, l'hypothèse de la présence d'une narratrice. De même, la simple compréhension de la différence séparant «niveau » et "personne» interdit de présenter l'instance qui assume la conduite de ce récit comme un narrateur intradiégétique. Mais, une fois écartées de telles bévues, le récit durassien n'en pose pas moins d'intéressants problèmes. Pour mémoire, le narrateur primaire, en tant que tel extradiégétique («vu?»...), n'apparaît tout d'abord pas très clairement comme personnage de l'histoire qu'il raconte, en raison notamment de son anonymat (initial). Mais cette situation parait soudainement évoluer aux environs du tiers ${ }^{57}$ de l'ouvrage, lorsqu'il révèle tardivement son identité, "Jacques Hold», avant de spécifier les liens qui l'unissent aux autres personnages de la diégèse. On pourrait donc éprouver l'impression d'une mutation diachronique de la relation de personne, un narrateur tout d'abord hétérodiégétique cédant la place à un narrateur homodiégétique. Mais une telle interprétation ferait bon marché de la lettre du texte. «Je n'ai rien entendu dire sur l'enfance de Lol V. Stein qui m’ait frappé, même par Tatiana Karl, sa meilleure amie durant leurs années de collège» (p. 11) : cette phrase de l'incipit suffit à établir un rapport de contiguïté entre le narrateur et l'univers spatio-temporel de l'histoire qu'il raconte, car où, sinon dans la diégèse, veut-on qu'il ait pu recueillir ces informations, même peu «frappantes», de la part d'un de ses personnages ${ }^{58}$ ? De même, affirmer comme il le fait «l'écrasante actualité de cette femme [Lol V. Stein] dans [sa] vie» (p. 14) constitue un indice supplémentaire, parmi bien d'autres, du rapport de phase qui l'unit à la diégèse et à ses «autochtones». Bref, ne pas percevoir ces phénomènes témoigne certes d'une forme de surdité critique, mais c'est la discrétion actantielle de l'instance bifrons que constitue ce narrateur-personnage (extra-homodiégétique) qui vient sans doute brouiller la perception de son statut; du moins pour un temps, jusqu’à la révélation par lui-même de son identité et de ses rapports aux autres personnages.

En regard des expérimentations entreprises par d'autres «nouveaux romanciers» en matière de traitement déceptif de la voix narrative ${ }^{59}$, l'audace du procédé durassien pourrait somme toute paraître timide; au «scandale» logique près qui en résulte. En effet, avant de révéler tardivement son identité, le narrateur-personnage s'est décrit, sans le signaler, suivi par Lol V. Stein, alors qu'il se promenait en compagnie de sa

55. C'est-à-dire le poids d'un «diktat» narratologique, dont il me semble que nous sommes désormais bien loin. Où sont les neiges d'antan?

56. "Je n'ai rien entendu dire [...] qui m’ait frappé [...]» (op. cit., p. 11, je souligne).

57. En fait, à la page 74 de l'édition que j’utilise, qui en compte 191: "Tatiana présente à Lol Pierre Beugner, son mari, et Jacques Hold, un de leurs amis, la distance est couverte, moi.» La «distance» ici évoquée désigne à la fois celle qui sépare les femmes de la terrasse où se tiennent les hommes, celle qui sépare Lol de Jacques Hold, celle qui sépare le narrateur du personnage.

58. A celles et ceux qui, sur ce point, demeureraient sceptiques, on conseillera de tenter d'interroger Tatiana Karl...

59. Que l'on pense par exemple à L'Innommable (Paris, éd. Minuit [1949], 1953) de Samuel Beckett... 
maîtresse, Tatiana $\operatorname{Karl}^{60}$; d'où, rétrospectivement, pour les lecteurs, l'impression d'avoir été victimes d'une forme de mensonge par omission. Du moins, ce peut être le cas pour qui ne prête pas une attention suffisante aux incidentes métatextuelles à valeur de "guide-âne», comme "Je crois voir ce qu'a dû voir Lol V. Stein" (p. 59), qui contribuent tout de même notablement à désambiguïser la situation narrative. Le reste est affaire d'interprétation: c'est manifestement sous le poids de son désir pour Lol que le narrateur-personnage est conduit à adopter les limitations cognitives de l'héroïne, partant à se présenter comme un simple quidam, et plus généralement à minorer l'importance de son rôle actantiel, aussi longtemps qu'il ne lui a pas été présenté. Cette substitution d'une logique fantasmatique d'ordre fusionnel à la logique narrative usuelle permet donc de résorber l'apparent scandale évoqué ci-dessus, comme de comprendre les fluctuations pronominales qui affectent nombre de scènes érotiques de la seconde "partie» du livre. En effet, la particularité majeure de ces séquences narratives, consistant en l'évocation des rendez-vous amoureux de Jacques Hold et Tatiana Karl, dans une chambre d'hôtel dont la fenêtre ouvre sur un champ de seigle où se dissimule Lol V. Stein, réside dans l'emploi indifférencié que fait le narrateur-personnage du «je» et du «il » pour se désigner lui-même. Si les deux pronoms apparaissent coréférents, c'est à la fois en raison du cadre diégétique (un huis-clos amoureux, impliquant un nombre réduit [deux] de personnages ${ }^{61}$ ) et de la logique fantasmatique déjà identifiée. On remarquera d'ailleurs que le pronom «je» est utilisé lors des mouvements du narrateur-personnage vers la fenêtre, donc vers Lol; le pronom «il» lors des mouvements inverses vers le lit, donc vers Tatiana ${ }^{62}$, "celle qui n'est pas son amour» (p. 134). En inférer que l'emploi du pronom «il» signale alors l'aliénation du narrateur-personnage ne devrait pas excéder les compétences interprétatives d'un lecteur attentif - l'esthétique du ressassement propre au récit durassien en sauvegardant, aussi sibyllin paraisse-t-il de prime abord, la lisibilité.

Quoi que l'on pense de Duras, de son œuvre et de ses topiques de prédilection, force est donc de convenir que le traitement hétérodoxe de la relation de personne dans Le Ravissement de Lol V. Stein mérite de retenir l'attention, en raison du trouble qui l'affecte, et peut, au moins momentanément, vouer les lecteurs à la perplexité. Pour rendre justice à l'écrivain, reste à préciser que cette anomie ne découle pas chez elle d'un parti pris hyperformaliste, mais de la quête inlassable du «moyen de dévoilement, d'exploration ${ }^{63}$ ", qui, sur fond de remise en cause des conceptions unitaires du sujet, la voue à l'élaboration d'une esthétique dysnarrative.

60. P. $52 s q$.

61. Sauf à supposer la présence d'un amant dans le placard; mais nous sommes chez Duras, pas chez Labiche ou Feydeau...

62. "Je suis retourné à la fenêtre, elle [Lol V. Stein] était toujours là, là dans ce champ d'une manière dont elle ne pouvait témoigner devant personne. J'ai su cela d'elle en même temps que j'ai su mon amour, sa suffisance inviolable, géante aux mains d'enfant. Il regagna le lit, s'allongea le long de Tatiana Karl. Ils s'enlacèrent dans la fraîcheur du soir naissant» (op. cit., p. 125).

63. Marguerite Duras, India Song, Paris, Gallimard, «Blanche», 1973; puis «L'Imaginaire», 1991, p. 10. 
Malgré certains refus partagés avec Duras, la posture d'auteur de Robbe-Grillet, on le sait, était sensiblement différente. Aussi est-ce sans surprise, mais non sans intérêt, que l'on peut découvrir dans son œuvre ce qui ressemble d'assez près à une variation littéraire sur la définition genettienne de la relation de personne. Tel est bien le cas dans La Reprise ${ }^{64}$, où deux narrateurs rivaux s'affrontent, le second (par ordre d'apparition) contestant la véracité de la narration du premier, à la faveur d'un inattendu dispositif notulaire. Qu'on en juge, à la lecture de ce fragment de la note 4:

Note 4 - Pas plus que le passage de la première à la troisième personne [...], ce remplacement impromptu de l'indicatif présent par le passé indéfini, d'ailleurs temporaire, ne modifie à notre sens ni l'identité du narrateur ni l'époque de la narration. Quelle que soit la distance que semble prendre la voix narrative par rapport au personnage, le contenu des énoncés ne cesse à aucun moment de reproduire une connaissance intérieure de soi-même, autoperceptive et instantanée [...]. Le point de vue reste bien toujours celui de notre sujet multinominal et volontiers pseudonyme [...] (p. 65).

Affirmation de la prévalence de l'attitude narrative sur les choix pronominaux, soulignement du rapport de contiguïté unissant le narrateur à l'histoire dont il assume la relation, lien au "point de vue ${ }^{65}$ » : tout y est. Ce commentaire métatextuel permet ainsi de dispenser en contrebande aux lecteurs une manière de cours de narratologie appliquée, que l'on se gardera pourtant de verser trop hâtivement au passif de quelque "formalisme gratuit»: dans La Reprise, les enjeux de la relation agonistique opposant les deux narrateurs sont de l'ordre de la survie - Schéhérazade n'est (décidément) pas morte ${ }^{66}$.

En dépit de la date de publication (2001) du roman, d'aucuns estimeront peut-être qu'avec La Reprise ne fait que perdurer l'esthétique du "Nouveau Roman», qui nous a en effet de longue date accoutumés à un traitement déceptif de la voix narrative, par exemple en faisant alterner relations hétéro- et homodiégétique à l'échelle d'un même récit, comme Claude Simon dans La Route des Flandres ${ }^{67}$ ou Histoire ${ }^{68}$ ? Qu'à cela ne tienne: il ne manque pas d'écrivains postérieurs à la mouvance néoromanesque pour inquiéter, chacun à sa façon, la relation de personne. A commencer par Antoine Volodine, l'inventeur du "post-exotisme», esthétique imaginaire dont l'indifférence aux distinctions pronominales constitue l'un des principes définitoires - comme l'atteste l'extrait suivant:

64. Paris, éd. de Minuit, 2001.

65. Pour des raisons d'économie, j'ai dû me résoudre à passer sous silence ces liens de la voix et du mode ("focalisation»), effectivement importants, comme l'attestait déjà le cas du Ravissement de Lol V. Stein. D’ailleurs, en termes d'effets, les similitudes entre narration homodiégétique préfocalisée et narration hétérodiégétique en focalisation interne sont indéniables. Sur la notion de situation narrative, résultant de l'articulation des deux paramètres en cause, on se reportera à Franz K. Stanzel, Narrative Situations in the Novel, op. cit., et à Gérard Genette, Nouveau Discours du récit, op. cit., chap. XvII, p. 77-89.

66. Voir de nouveau Florian Pennanech, «La mort du narrateur (ou pas)», art. cité, p. 6-7.

67. Paris, éd. de Minuit, 1960.

68. Paris, éd. de Minuit, 1967 (prix Médicis). 
Je dis «je», «je crois» mais on aura compris qu'il s'agit, là aussi, de pure convention. La première personne du singulier sert à accompagner la voix des autres, elle ne signifie rien de plus. [...] Pour un narrateur post-exotique, de toute façon, il n’y a pas l'épaisseur d'une feuille de papier à cigarette entre la première personne et les autres, et guère de différence entre vie et mort $^{69}$.

Sans doute cette atténuation de la pertinence des distinctions pronominales ne constitue-t-elle qu'un épiphénomène quand on l'envisage à l'aune d'une esthétique aussi singulière, fondée sur une plus générale annulation du principe d'opposition des contraires; il n'empêche qu'elle informe massivement l'écriture des textes, et n'est pas sans incidence sur leur réception, puisque les lecteurs sont alors voués, bon gré mal gré, à ne plus pouvoir considérer l'opposition récit "personnel » vs «impersonnel» comme un repère stable sur lequel ils pourraient prendre appui pour identifier avec assurance l'origine de la parole narrative ${ }^{70}$.

Mais c'est sans doute dans La Femme changée en bûche $e^{71}$ de Marie NDiaye que le traitement anomique de la relation de personne est porté à son comble. Ce roman est en effet subdivisé en trois parties, la première conduite en relation homodiégétique, la deuxième en relation hétérodiégétique, quand la troisième multiplie les narrations homo- et hétérodiégétiques, parfois à l'intérieur d'une même phrase ${ }^{72}$. Est ainsi illustrée l'indéniable possibilité, pour un texte, de se situer «à cheval» sur la frontière censée séparer les relations de personne ${ }^{73}$, même s'il est évident qu'une configuration aussi tératologique n'est pas sans danger en matière de lisibilité.

Il faut également mentionner Nous trois ${ }^{74}$ de Jean Echenoz, roman plus discrètement, mais de façon non moins décisive, construit sur les flottements de la relation de personne. Si ce récit semble reposer sur l'alternance d'une narration hétérodiégétique (quantitativement majoritaire) et d'une narration homodiégétique (assumée par un certain «DeMilo»), la convergence finale de ces deux lignes d'histoire, d'abord en apparence autonomisées, engendre une forme hybride éminemment problématique, qu'il est dès lors extrêmement délicat d'interpréter. En effet, à partir du chapitre 23 (ou plus nettement 24), on peut soit estimer que perdure une narration hétérodiégétique dominante, mais "parasitée» par des îlots de narration homodiégétique; soit, à l'inverse - et plus probablement, me semble-t-il -, opter pour

69. Le Post-exotisme en dix leçons, leçon onze, Paris, Gallimard, «Blanche», 1998, p. 19.

70. Sur la question de la voix narrative dans l'œuvre post-exotique, qu'on me permette de renvoyer à Frank Wagner, «Les voix du post-exotisme (sont-elles impénétrables?) ", Vox-poetica, en ligne, 15 avril 2014, URL: http://www.vox-poetica.org/t/articles/wagner2014.html

71. Paris, éd. de Minuit, 1989.

72. En fait, l'extrême singularité de ce récit implique selon moi de procéder à une étude croisée de la voix et du mode. C'est ce que j'avais tenté de réaliser dans un article intitulé «Parler et percevoir. Les fluctuations de la situation narrative dans La Femme changée en bûche de Marie NDiaye», Poétique, $n^{\circ} 150$, février 2007, p. 217-237. Le cas échéant, on voudra bien s'y reporter.

73. Ce qui m'incite à me rallier à la position exposée par Genette dans Nouveau Discours du récit, op. cit., p. 70, contre celle que défend Dorrit Cohn dans «The Encirclement of Narrative», Poetics Today, 2, 2, hiver 1981, p. 168.

74. Paris, éd. de Minuit, 1992. 
l'hypothèse d'une narration homodiégétique dont l'instance se ferait simplement plus discrète lorsqu' elle évoque les agissements des autres personnages. En outre, sur cette base (hypothèse $\mathrm{n}^{\circ} 2$ ), peut naître rétrospectivement l'intuition que DeMilo était en fait narrateur (homodiégétique) depuis le début du roman, et qu'il s'était dissimulé à des fins manipulatrices durant les chapitres en apparence hétérodiégétiques - hypothèse que contrarierait toutefois la dimension paraleptique des sections conduites «à la troisième personne ${ }^{75}$ ». Dans le doute, je mabstiendrai de trancher entre ces deux lectures possibles, l'essentiel résidant en l'occurrence dans l'indiscutable flottement qui affecte la relation de personne. Il s'agit d'ailleurs là d'une caractéristique beaucoup plus générale de l'œuvre d'Echenoz, qui pose avec acuité la question du statut de l'instance narrative.

Enfin, aux confins de la fiction ${ }^{76}$, dans son dernier ouvrage en date, Aurais-je sauvé Geneviève Dixmer?, Pierre Bayard se livre, sur fond de métalepse (puisqu'il se substitue au personnage de Maurice Lindey), pour accentuer la portée de cet essai de philosophie morale appliquée à la littérature (ou est-ce l'inverse?...), à un exercice de transvocalisation en relation homodiégétique de nombreux extraits du Chevalier de Maison-Rouge 77 d'Alexandre Dumas. On constate donc que Jean-Philippe Toussaint se trouve en définitive en bonne (et abondante) compagnie.

Quels enseignements tirer de ce parcours tripartite? En dépit de menus flottements (notamment diachroniques), expliquant pour partie les désaccords de certains autres poéticiens, la relation de personne telle que la définit Genette parait à la fois cohérente et opératoire - ce qui suffit à mes yeux à la dédouaner des accusations d'obsolescence qui ont pu être formulées à son encontre ${ }^{78}$. Disons, pour l'essentiel, que la prévalence de l'attitude narrative sur les choix grammaticaux (pronominaux) est aussi bien valide sur le plan théorique qu'attestée sur le plan artistique; de même que la possibilité de situations mixtes ou ambiguës; ou encore que les difficultés de distinction entre les types - l'avantage d'une poétique ouverte étant qu'elle ne risque pas de se voir invalider par les cas d'espèce...

Pour autant, on ne peut nier que certains des troubles précédemment évoqués soient appelés à perdurer, et à alimenter encore bien des débats, sans doute. Ainsi, en particulier, de la question de savoir s'il est ou non nécessaire de recourir à la notion de narrateur en régime d'hétérodiégéticité. Aussi longtemps que l'on s’inscrit, comme je

75. Même si une première paralepse spectaculaire est présente à la fin du chapitre I (p. 11), conduit en narration homodiégétique, préparant ainsi le terrain pour celles qui suivront, dans les chapitres en relation hétérodiégétique. On pourrait donc estimer que l'extrait de la page 11 s'approche d'une forme de «règle du jeu ", certes éminemment paradoxale, et en tant que telle sujette à interprétation(s).

76. De ces "confins» relèverait également la forme par essence hybride que l'on nomme "autofiction", et dont on connaît la vogue à l'époque contemporaine. Ce genre (si c'en est un) repose en effet sur l'établissement de relations paradoxales entre auteur, narrateur et personnage. Mais l'ambiguïté des pratiques autofictionnelles est telle qu'on comprendra peut-être que je ne les aborde pas ici, où la place m'est comptée.

77. (1845-1846), Paris, Gallimard, «Folio», 2005, pour l'édition citée par Bayard.

78. Par Sylvie Patron, dans Le Narrateur, op. cit., en particulier p. 9. En fait, les «accusations d'obsolescence" y concernent Nouveau Discours du récit dans son ensemble. 
l'ai fait, dans une perspective narratologique, la réponse à cette question ne peut être qu'affirmative - puisque la dissociation des instances littéraire et narrative constitue l'un des fondements de la discipline. Mais dès lors que l'on aborde le problème de plus loin, en l'envisageant en termes de statut logique de l'activité linguistique productrice des énoncés fictionnels, comme l'a fait Genette dans Fiction et diction, où il se livre à une réappropriation personnelle de la théorie des actes de langage de John Searle 79 , les divergences d'opinions paraissent inévitables. Toutefois, reprocher à Genette, parmi d'autres narratologues, d'avoir «mal compris ${ }^{80}$ » les travaux en question n'a guère de sens, puisque son objectif n'est ni d'obtenir un diplôme de philosophie du langage ni de mériter l'approbation de l'auteur de Sens et expression, mais de tenter, en réactualisant cette pensée, de déterminer, dans une perspective poétologique, «la meilleure, ou la moins mauvaise, façon de dire ce que fait l'auteur du Père Goriot» (Fiction et diction, p. 46), et non "pas exactement de savoir si les énoncés constitutifs du récit de fiction sont ou non des actes illocutoires" (idem).

Mais qu'en est-il de la relation de personne reconsidérée dans cette perspective ${ }^{81}$ ? Le type homodiégétique relève alors d'une substitution d'identité narrative, puisque l'auteur (Doyle) feint d'être quelqu'un d'autre (Watson) se livrant à des assertions tout à fait sérieuses dans l'univers fictionnel; quand, dans le type hétérodiégétique, l'auteur produit des assertions feintes ou simule des actes illocutoires ${ }^{82}$. Ainsi présentée, cette distinction pourrait sembler problématique, puisque, en relation d'hétérodiégéticité, l'attribution de la feintise à l'auteur paraît entraîner la disparition du narrateur. Genette devance toutefois cette objection en ces termes:

[...] dire, comme Searle, que l'auteur (par exemple, Balzac) ne répond pas sérieusement des assertions de son récit (par exemple, l'existence d'Eugène Rastignac), ou dire que nous devons les rapporter à une instance implicite distincte de lui (le narrateur du Père Goriot), c'est dire la même chose de deux manières différentes, entre lesquelles seul le principe d'économie nous fait choisir, selon les nécessités du moment (Fiction et diction, p. 80-81).

Tout le problème est d'établir s'il y a bien là relation tautologique, c'est-à-dire simple différence de formulation. Pour ma part, j’incline à le penser, sans pour autant dédaigner a priori les opinions contraires, pour peu qu'elles reposent sur des arguments plus convaincants qu'un constat d'hétérodoxie à l'égard de la pensée de Searle - ou que des accusations de mécompréhension. A l'image de la poétique

79. Les Actes de langage. Essai de philosophie du langage (1969), Paris, Hermann, 1972 pour la traduction française; Sens et expression (1979), Paris, éd. de Minuit, «Sens commun», 1982, pour la traduction française.

80. Sylvie Patron, Le Narrateur, op. cit., p. 99: «[...] les narratologues [Lubomir Doležel, MarieLaure Ryan et Gérard Genette] comprennent mal la position des philosophes, de Searle en particulier, sur certains points cruciaux. Une partie des faiblesses internes de leur argumentation s'explique par ces incompréhensions.»

81. Sur cette question, voir en particulier, dans Fiction et diction, op. cit., le chapitre intitulé «Les actes de fiction» (p. 41-63).

82. Cette autre formulation est empruntée à Jean-Marie Schaeffer, Pourquoi la fiction?, Paris, éd. du Seuil, «Poétique», 1999, p. 255 et passim. 


\section{Frank Wagner}

genettienne, le débat reste donc ouvert. A tout le moins, ces désaccords, passés, présents et (très probablement) futurs confirment-ils, sur un autre plan, l'intuition à l'origine de cette enquête: celle de troubles dans la relation de personne.

Université Rennes 2 